East Asian Mathematical Journal

Vol. 29 (2013), No. 1, pp. 69-82

http://dx.doi.org/10.7858/eamj.2013.006

\title{
DOUBLY NONLINEAR VOLTERRA EQUATIONS INVOLVING THE LERAY-LIONS OPERATORS
}

\author{
KIYEON SHIN* AND SUJIN KanG
}

\begin{abstract}
In this paper we consider a doubly nonlinear Volterra equation related to the Leray-Lions with a nonsmooth kernel. By exploiting a suitable implicit time-discretization technique we obtain the existence of global strong solution.
\end{abstract}

\section{Introduction}

In this paper, we study a doubly nonlinear Volterra partial differential equation involving the Leray-Lions operator. More precisely, we are interested in the existence and uniqueness of the solution of problem

$$
\begin{aligned}
& \frac{\partial \beta(u)}{\partial t}-\operatorname{div}[\mathrm{a}(\mathrm{x}, \mathrm{u}, \nabla \mathrm{u})+\mathrm{k} * \mathrm{a}(\mathrm{x}, \mathrm{u}, \nabla \mathrm{u})] \\
& +f(x, t, u)=0 \quad \text { in } \Omega \times[0, T], \\
& u=0 \\
& \text { on } \partial \Omega \times[0, T] \text {, } \\
& u=u^{0} \\
& \text { on } \Omega \times\{t=0\} \text {, }
\end{aligned}
$$

where $-\operatorname{div}[\mathrm{a}(\mathrm{x}, \mathrm{u}, \nabla \mathrm{u})]=\mathrm{Au}$ is the Leray-Lions operator, $k \in B V(0, T), \beta$ is a nonlinearity of porous medium type and $f$ is a nonlinearity of reaction diffusion type. A prime example of $\operatorname{div}[a(x, u, \nabla u)]$ is $p$-Laplacian $\Delta_{p} u=$ $\operatorname{div}\left[|\nabla u|^{p-2} \nabla u\right], 2 \leq p<\infty$. The convolution sign has to be understood in sense of the standard product in $(0, t) \subset(0, T)$ where $T>0$ denotes some reference final time. In particular, $(a * b)(t)=\int_{0}^{t} a(t-s) b(s) d s$ whenever it makes sense. Let $\Omega$ be a regular open bounded set of $\mathbb{R}^{d}, d \geq 1$ and $\partial \Omega$ its boundary.

The problem (1) has a relevant interest within applications since it arises in nonlinear diffusion phenomena including nonlocal time effect. For example, we consider a substance which fills the region $\Omega \subset \mathbb{R}^{3}$ and may undergo a temperature driven phase transformation. We assume that our thermodynamic

Received December 6, 2012; Accepted January 9, 2013.

2000 Mathematics Subject Classification. 45K05, 35K55, 35K45.

Key words and phrases. Doubly nonlinear, volterra equation, discretization, leray-lions operator.

This work was supported by a 2-Year Research Grant of Pusan National University.

*Corresponding author. 
system is insulated from the exterior and fix as state variable the (relative) temperature $\theta$ of the medium. By the energy balance relation, we have the equation

$$
e_{t}+\operatorname{div}[q]=g \quad \text { in } \quad \Omega \times(0, T)
$$

where $e$ is the internal energy of system (enthalpy), $q$ is the heat flux and $g$ is a given density of heat source.

Since the heat flux is the datum of an actual contribution $\left(k_{0}>0\right.$ represents an instantaneous heat conductivity) and an accumulated history averaged by means a suitable kernel $K_{1}$, we choose the following

$$
q(t)=k_{0}|\nabla \theta|^{p-2} \nabla \theta(t)-\int_{0}^{t} K_{1}(t, s) \nabla \theta(s) d s .
$$

Therefore, the equation becomes one of type (1).

Let us now try to give brief comment on the current literature for doubly nonlinear Volterra equations of the type (1). Of course the local-in-time case $k=0$ in (1) has been deeply discussed by Alt and Luckhaus [2], J.I. Diaz and J.F. Padial [5], A. Eden, B. Michaux and J.M. Rakotoson [6, 7], A. El Hachimi and H. El Ouardi [8], Ivanov and Rodrigues [10], Otto [11], A. Rougirel [12], M. Schatzman [13] and K. Shin and S. Kang [14] in various methods. For the nonlocal case $k \neq 0$, Stefanelli [17] studied in case of $\beta=I,-\operatorname{div}[a(x, u, \nabla u)]=$ $-\Delta u$ and $k \in W^{1,1}(0, T)$ in Hilbert space. Also, [18] is studied where $\beta$ is maximal monotone, $k \in L^{1}(0, T)$ and $f \in L^{q}\left(0, T ; V^{*}\right)$ where $q$ is conjugate of $p$ and $V^{*}$ is dual of reflexive Banach space. Moreover, when $\beta$ is maximal monotone and $k \in B V(0, T)$ with $f \in L^{q}\left(0, T ; V^{*}\right)$, it has been considered by Gilardi and Stefanelli [9].

This is the plan of paper. We recall our assumptions and state main results in section 2. In section 3, we show the existence of discrete scheme. After showing some estimates on the approximations, the passage to the limit and the existence results are given in section 4.

\section{Assumptions and main result}

We let $\|\cdot\|_{p},\|\cdot\|_{1, p},\|\cdot\|_{-1, p}$ and $\|\cdot\|_{X}$ denote the norm in $L^{p}(\Omega), W^{1, p}(\Omega)$, $W^{-1, p}(\Omega)$ and $X(1 \leq p \leq \infty)$, respectively. $<\cdot, \cdot>$ denotes the duality between $W_{0}^{1, p}(\Omega)$ and $W^{-1, p^{\prime}}(\Omega)$ or inner product of $L^{2}(\Omega)$. For $p \geq 2$, we define $p^{\prime}$ by $\frac{1}{p}+\frac{1}{p^{\prime}}=1$. In this paper, $C_{i}$ and $C$ will denote positive constants and $\lambda_{1}, \lambda_{2}$ imbedding constants. (cf. [1])

Let $u^{0} \in L^{\infty}(\Omega)$ and $\beta$ be a continuous function with $\beta(0)=0$. For $t \in \mathbb{R}$, define $\psi(t)=\int_{0}^{t} \beta(s) d s$. The Legendre transform of the convex function $\psi$ is defined as $\psi^{*}(\tau)=\sup _{s \in \mathbb{R}}\{\tau s-\psi(s)\}$.

Now, we prepare assumptions, well known definitions and lemmas which have used throughout this paper. 
First of all, for any $u \in L^{1}(0, T)$, let us set

$$
\operatorname{Var}(u):=\sup \left\{\int_{0}^{T} u \varphi^{\prime} \mid \varphi \in C_{0}^{1},\|\varphi\|_{L^{\infty}(0, T)} \leq 1\right\}
$$

and

$$
B V(0, T):=\left\{u \in L^{1}(0, T) \mid \operatorname{Var}(u)<\infty\right\} .
$$

The latter turns out to be a Banach space whenever endowed with the norm

$$
\|u\|_{B V(0, T)}:=\|u\|_{1}+\operatorname{Var}(u) .
$$

For all $u \in B V(0, T)$, there exist a right-continuous function $\widetilde{u}$ such that $\widetilde{u}=u$ almost everywhere in $(0, T)$ and

$$
\operatorname{Var}(u)=\operatorname{Var}(\widetilde{u})=\sup \left\{\sum_{i=2}^{N}\left|\widetilde{u}\left(t_{i}\right)-\widetilde{u}\left(t_{i-1}\right)\right| \text { for } 0<t_{1}<\cdots<t_{N}<T\right\} .
$$

One should notice that $\widetilde{u}$ is bounded and can be represented as the difference two (bounded) monotone functions. In particular, $\widetilde{u}$ turns out to admit right (left) limit in $0(T)$, respectively. Hence, by defining $\widetilde{u}(0):=\widetilde{u}\left(0_{+}\right)$and $\widetilde{u}(T):=$ $\widetilde{u}\left(T_{-}\right)$with obvious notions, one readily has that

$$
\operatorname{Var}(\widetilde{u})=\sup \left\{\sum_{i=1}^{N}\left|\widetilde{u}\left(t_{i}\right)-\widetilde{u}\left(t_{i-1}\right)\right| \text { for } 0=t_{0}<\cdots<t_{N}=T\right\},
$$

as well.

We suppose that $u^{0} \in L^{\infty}(\Omega)$ with $u^{0}=0$ on $\partial \Omega$ and the followings ;

(H1) The function $\beta$ is increasing and continuous from $\mathbb{R}$ to $\mathbb{R}$ and $\beta(0)=0$.

(H2) $a(x, s, \xi)$ is a Caratheodory function $a: \Omega \times \times \mathbb{R}^{d} \rightarrow \mathbb{R}^{d}$ such that

$$
\begin{aligned}
& |a(x, s, \xi)| \leq \gamma\left[|s|^{p-1}+|\xi|^{p-1}+l(x)\right], \\
& {[a(x, s, \xi)-a(x, s, \eta)](\xi-\eta)>0, \text { for } \xi \neq \eta,} \\
& a(x, s, \xi) \xi \geq \alpha|\xi|^{p},
\end{aligned}
$$

where $l \in L^{p^{\prime}}(\Omega), l \geq 0, \gamma>0$ and $\alpha>0$.

(H3) For $\xi \in \mathbb{R}$, the map $(x, t) \mapsto f(x, t, \xi)$ is measurable and $\xi \mapsto f(x, t, \xi)$ is continuous a.e. in $\Omega \times[0, T]$. Furthermore, we assume that there exits $C_{1}>0$ such that $\operatorname{sign} \xi f(x, t, \xi) \geq-C_{1}$ for a.e. $(x, t) \in \Omega \times[0, T]$ and there exits $C_{2}>0$ such that $\xi \mapsto f(x, t, \xi)+C_{2} \beta(\xi)$ is increasing for almost $(x, t) \in \Omega \times[0, T]$.

(H4) For all $M>0$, there exists $C_{M}>0$ such that, if $|\xi|+\left|\xi^{\prime}\right| \leq M$ then

$$
\left|f(x, t, \xi)-f\left(x, t, \xi^{\prime}\right)\right|^{p^{\prime}} \leq C_{M}\left(\beta(\xi)-\beta\left(\xi^{\prime}\right)\right)\left(\xi-\xi^{\prime}\right) .
$$

(H5) For almost every $x \in \Omega$ and for all $M>0$, there exists $\widetilde{C}_{M}>0$ such that, if $t+t^{\prime}+|\xi| \leq M$ then

$$
\left|f(x, t, \xi)-f\left(x, t^{\prime}, \xi\right)\right| \leq \widetilde{C}_{M}\left|t-t^{\prime}\right|^{1 / p^{\prime}} .
$$

(H6) $k \in B V(0, T)$. 
Definition 1. ([3]) Let $X$ be a reflexive Banach space and $A: X \rightarrow X^{\prime}$ be an operator. We say that $A$ is monotone if $\langle A y-A z, y-z\rangle \geq 0 \forall y, z \in X$, and hemicontinuous if for each $y, z, w \in X$ the real-valued function $t \rightarrow\langle A(y+$ $t z), w\rangle$ is continuous.

Lemma 2.1. (Minty theorem [15]) If $X$ is a reflexive Banach space and $A$ : $X \rightarrow X^{\prime} X$ is monotone and hemicontinuous, then

$$
A y=f \text { if and only if }\langle f-A z, y-z\rangle \geq 0 \text { for all } z \in X \text {. }
$$

Lemma 2.2. ([4]) Let $\Omega$ be a bounded set in $\mathbb{R}^{d}$. Let $1<p<\infty$ be fixed and $A: W_{0}^{1, p}(\Omega) \rightarrow W^{1, p^{\prime}}(\Omega)$ be a nonlinear operator defined by

$$
A(u)=-\operatorname{div} a(x, u, D u)
$$

where $a(x, s, \xi)$ is a Carathéodory function $a: \Omega \times \mathbb{R} \times \mathbb{R}^{d} \rightarrow \mathbb{R}^{d}$ such that

$$
\begin{aligned}
& |a(x, s, \xi)| \leq \gamma\left[|s|^{p-1}+|\xi|^{p-1}+l(x)\right], \\
& {[a(x, s, \xi)-a(x, s, \eta)](\xi-\eta)>0, \quad \xi \neq \eta,} \\
& a(x, s, \xi) \xi \geq \alpha|\xi|^{p},
\end{aligned}
$$

where $l(x) \in L^{p^{\prime}}(\Omega), l \geq 0, \gamma>0$ and $\alpha>0$.

Let $g(x, s, \xi)$ be a Carathéodory function such that

$$
\begin{aligned}
& g(x, s, \xi) s \geq 0, \\
& |g(x, s, \xi)| \leq b(|s|)\left(|\xi|^{p}+c(x)\right),
\end{aligned}
$$

where $b$ is a continuous and increasing function with (finite) values on $\mathbb{R}^{+}$and $c \in L^{1}(\Omega), c \geq 0$. Then, for $h \in W^{-1, p^{\prime}}(\Omega)$, the problem

$$
A u+g(x, u, \nabla u)=h,
$$

has at least one solution $u \in W_{0}^{1, p}(\Omega)$.

The main theorem is the following.

Theorem 2.3. Under assumptions $(H 1)-(H 6)$, there exist $u \in L^{p}\left(0, T ; W_{0}^{1, p}(\Omega)\right)$ fulfilling (1) if $p \geq 2$.

\section{Time-discretization}

Let us start by fixing a uniform partition of the time interval $[0, T]$ by choosing a constant time-step $\tau=T / N, N \in \mathbb{N}$.

\subsection{Discrete convolution and Approximation of the kernel}

Definition 2. ([9]) Let $a=\left\{a_{i}\right\}_{i=1}^{N}$ be a real vector and $b=\left\{b_{i}\right\}_{i=1}^{N} \in$ $E^{N}$, where $E$ stands for a real linear space. Then, we define the vector $\left\{\left(a *_{\tau} b\right)_{i}\right\}_{i=0}^{N} \in E^{N+1}$ as

$$
\left(a *_{\tau} b\right)_{i}:= \begin{cases}0, & \text { if } \quad i=0, \\ \tau \sum_{j=1}^{i} a_{i-j+1} b_{j}, & \text { if } \quad i=1,2, \ldots, N .\end{cases}
$$


Let us list some properties of the latter discrete convolution product. First of all, we readily check that, for all $a=\left\{a_{i}\right\}_{i=1}^{N}, b=\left\{b_{i}\right\}_{i=1}^{N} \in \mathbb{R}^{N}$ and $c=\left\{c_{i}\right\}_{i=1}^{N} \in E^{N}$, one has

$$
\left(a *_{\tau} b\right)=\left(b *_{\tau} a\right), \quad\left(\left(a *_{\tau} b\right) *_{\tau} c\right)=\left(a *_{\tau}\left(b *_{\tau} c\right)\right) .
$$

In the forthcoming discussion, the following notations will be used extensively. Letting $\left\{u_{i}\right\}_{i=0}^{N}$ be vector, we denote by $u_{\tau}$ and $\bar{u}_{\tau}$ two functions on the time interval $[0, T]$ which interpolate the values of the vector $\left\{u_{i}\right\}_{i=0}^{N}$ piecewise linearly and backward constantly on partition of diameter, respectively. Namely,

$$
\begin{array}{ll}
u_{\tau}(0):=u_{0}, & u_{\tau}(t):=u_{i}+\frac{u_{i}-u_{i-1}}{\tau}(t-i \tau), \\
\bar{u}_{\tau}(0):=u_{0}, & \bar{u}_{\tau}(t):=u_{i}=u(\cdot, i \tau)
\end{array}
$$

for $t \in((i-1) \tau, i \tau], i=1,2, \ldots, N$. Let us also set $\bar{f}_{\tau}(t):=f_{i}=f\left(\cdot, i \tau, u_{i}\right)$ for $t \in((i-1) \tau, i \tau], v_{i}=\beta\left(u_{i}\right)$ and $\delta u_{i}=\frac{u_{i}-u_{i-1}}{\tau}$ for $i=1,2, \ldots, N$. Then, of course $\delta u$ stands for the vector $\left\{\delta u_{i}\right\}_{i=1}^{N}$ owing to the previous notation. It is not difficult to check the following equality holds.

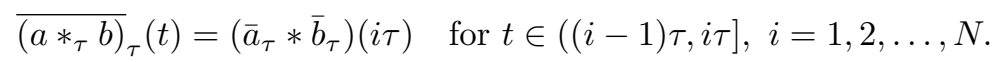

Moreover, the function $\bar{a}_{\tau} * \bar{b}_{\tau}$ is piecewise affine on the time partition. The reader should note that the above discussion yields. In particular,

$$
\left(a *_{\tau} b\right)_{\tau}=\bar{a}_{\tau} * \bar{b}_{\tau} \quad \text { in }[0, T] .
$$

Now, we recall a discrete version of Young's theorem and properties which are needed in the following.

Lemma 3.1. (Discrete Young theorem [17]) Let $\left\{a_{i}\right\}_{i=0}^{N} \in \mathbb{R}^{N},\left\{b_{i}\right\}_{i=1}^{N} \in E^{N}$, where $E$ denotes a real linear space endowed with the norm $\|\cdot\|_{E}$. Moreover, let $p, q, r \in[1, \infty]$ such that

$$
1+\frac{1}{r}=\frac{1}{p}+\frac{1}{q}
$$

along with the standard convention $1 / \infty=0$. Then the following inequality holds

$$
\left\|{\overline{\left(a *_{\tau} b\right)}}_{\tau}\right\|_{L^{r}(0, T ; E)} \leq\left\|\bar{a}_{\tau}\right\|_{L^{p}(0, T ; E)}\left\|\bar{b}_{\tau}\right\|_{L^{q}(0, T ; E)} .
$$

With help of Lemma 3.1, we have the following.

Proposition 3.2. ([9]) Let $r \in[1, \infty],\left\{a_{i}\right\}_{i=0}^{N} \in \mathbb{R}^{N+1}$ and $\left\{b_{i}\right\}_{i=1}^{N} \in E^{N}$, where $E$ denotes a real Banach space. Then, we have

$$
\left\|{\overline{\left(a *_{\tau} b\right)}}_{\tau}-\bar{a}_{\tau} * \bar{b}_{\tau}\right\|_{L^{r}(0, T ; E)} \leq \tau C_{r}\left(\operatorname{Var}\left(a_{\tau}\right)+\left|a_{0}\right|\right)\left\|\bar{b}_{\tau}\right\|_{L^{r}(0, T ; E)},
$$

where $C_{r}:=(1+r)^{-1 / r}$, for $r \in[1, \infty)$ and $C_{\infty}:=1$.

Using Lemma 3.1 and Proposition 3.2, we have very useful result by suitable passing to limits within discrete convolution products. 
Theorem 3.3. ([9]) Let $r \in[1, \infty]$ and $E$ be a real reflexive Banach space. If $\bar{a}_{\tau} \rightarrow$ a strongly in $L^{1}(0, T), a_{\tau}$ are equibounded in $B V(0, T)$, and $\bar{b}_{\tau} \rightarrow b$ weakly star (strongly) in $L^{r}(0, T ; E)$, then ${\overline{\left(a *_{\tau} b\right)}}_{\tau} \rightarrow a * b$ weakly star (strongly, respectively) in $L^{r}(0, T ; E)$.

Let us restrict ourselves to the case of a kernel $k:[0, T] \rightarrow \mathbb{R}$ such that

$$
\operatorname{Var}(k)=\sup \left\{\sum_{i=0}^{N}\left|k\left(t_{i}\right)-k\left(t_{i-1}\right)\right| \text { for } 0=t_{0}<\cdots<t_{N}=T\right\} .
$$

and

$$
k_{i}:=k(i \tau) \text { for } i=0,1, \ldots, N,
$$

whence it is a standard matter to verify that

$$
\left\|k-\bar{k}_{\tau}\right\|_{L^{1}(0, T)} \leq \operatorname{Var}(k) .
$$

Moreover, we readily check that

$$
\left\|k_{\tau}\right\|_{C[0, T]} \leq\|k\|_{L^{\infty}(0, T)} \text { and } \operatorname{Var}\left(k_{\tau}\right) \leq \operatorname{Var}(k),
$$

independently of $\tau$. For notational convenience, we will use the same symbol for the function $k$ and the vector $k=\left\{k_{i}\right\}_{i=0}^{N}$ whenever the latter is involved in a discrete convolution product. We shall look for a vector $\left\{\rho_{i}\right\}_{i=0}^{N} \in \mathbb{R}^{N+1}$ such that

$$
\rho_{i}+\left(k *_{\tau} \rho\right)_{i}=k_{i} \text { for } i=0,1, \ldots, N .
$$

The latter linear system may be solved whenever $\tau$ is small enough. Namely, $\rho_{0}=k_{0}=k(0)$, it is straightforward to check that the remaining $N \times N$ linear system is lower-triangular and its determinant reads $\left(1+\tau k_{1}\right)^{N}$. Hence, the latter is solvable whenever we have, for instance,

$$
\tau\left|k_{1}\right| \leq \frac{1}{2}
$$

which, taking into account (H5) and definition (2), holds at least for small $\tau$. We shall collect some properties of $\rho$ in the following proposition.

Proposition 3.4. ([9]) Let (H6) and (4) hold and $\left\{\rho_{i}\right\}_{i=0}^{N} \in \mathbb{R}^{N+1}$ be defined as above. Then

$$
\begin{aligned}
& \rho_{\tau} \text { are uniformly bounded in } B V(0, T) \text { in terms of }\|k\|_{B V(0, T)}, \\
& \left\|\rho_{\tau}-\bar{\rho}_{\tau}\right\|_{L^{1}(0, T)}=\frac{\tau}{2} \operatorname{Var}\left(\rho_{\tau}\right), \\
& \rho_{\tau} \rightarrow \rho \text { strongly in } L^{1}(0, T), \rho \in B V(0, T), \\
& \text { and } \rho+k * \rho=k \text { a.e. } \operatorname{in}(0, T) .
\end{aligned}
$$

Finally, owing to (3), we readily check that, given $\left\{a_{i}\right\}_{i=1}^{N},\left\{b_{i}\right\}_{i=1}^{N} \in E^{N}$ where $E$ is some real linear space,

$$
a_{i}+\left(k *_{\tau} a\right)_{i}=b_{i} \Leftrightarrow b_{i}-\left(\rho *_{\tau} b\right)_{i}=a_{i} \text { for } i=1, \ldots, N .
$$


Hence, let us conclude that, for all $a, b \in L^{1}(0, T: E)$, and $\left\{a_{i}\right\}_{i=1}^{N},\left\{b_{i}\right\}_{i=1}^{N} \in$ $E^{N}$, we have the following

$$
\begin{gathered}
a+(k * a)=b \text { a.e. in }(0, T) \Leftrightarrow b-(\rho * b)=a \text { a.e. in }(0, T), \\
\bar{a}_{\tau}+{\overline{\left(k *_{\tau} a\right)_{\tau}}}=\bar{b}_{\tau} \text { a.e. in }(0, T) \Leftrightarrow \bar{b}_{\tau}-{\overline{\left(\rho *_{\tau} b\right)_{\tau}}}=\bar{a}_{\tau} \text { a.e. in }(0, T) .
\end{gathered}
$$

\subsection{Existence of Discrete scheme}

For (1), we consider the discrete scheme (DS) for $i=1,2, \ldots, N$,

$$
\begin{array}{lr}
\frac{\beta\left(u_{i}\right)-\beta\left(u_{i-1}\right)}{\tau}-\operatorname{div} a\left(u_{i}, \nabla u_{i}\right)-\left(k *_{\tau} \operatorname{div} a(u, \nabla u)\right)_{i}+f\left(x, i \tau, u_{i}\right)=0, & \text { in } \Omega, \\
v_{i}=\beta\left(u_{i}\right) & \text { in } \partial \Omega, \\
u_{i}=0 & \text { in } \Omega .
\end{array}
$$

where $N \tau=T, T$ a fixed positive real. We shall show that (DS) has a solution $u_{i}, i=1,2, \ldots, N$.

Theorem 3.5. Assume that $(H 1)-(H 3),(H 6)$ and $(4)$ holds. Then for $i=$ $1,2, \ldots, N$, there exists a unique solution $u_{i} \in W_{0}^{1, p}(\Omega)$ of $(D S)$ for sufficiently small $\tau$.

Proof. First of all, we rewrite (DS) as

$$
-\tau\left(1+\tau k_{1}\right) \operatorname{div} a\left(u_{i}, \nabla u_{i}\right)+F\left(x, u_{i}\right)=\varphi_{i-1},
$$

where $F\left(x, u_{i}\right)=\beta\left(u_{i}\right)+\tau f\left(x, i \tau, u_{i}\right)+\tau C_{1} \operatorname{sign}\left(u_{i}\right)$ and $\varphi_{i-1}=\beta\left(u_{i-1}\right)+$ $\tau C_{1} \operatorname{sign}\left(u_{i}\right)+\tau^{2} \sum_{j=1}^{i-1} k_{i-j+1} \operatorname{div} a\left(u_{j}, \nabla u_{j}\right)$.

Now, we consider the equation

$$
-\tau\left(1+\tau k_{1}\right) \operatorname{div} a(u, \nabla u)+F(x, u)=\varphi_{0}=\beta\left(u_{0}\right)+\tau C_{1} \operatorname{sign}(u),
$$

where $F(x, u)=\beta(u)+\tau f(x, \tau, u)+\tau C_{1} \operatorname{sign}(u)$ for fixed $\tau=T / N$. It is obvious that $a(x, u, D u)$ satisfies all the three conditions of $a$ in Lemma 2.2 , by (4). Since $\beta$ is continuous, $\varphi_{0} \in L^{\infty}(\Omega)$. And, by (H1) and (H3), $g(x, u, \nabla u):=F(x, u)$ is a Carathéodory function with $u F(x, u) \geq 0$. Also, by (H3), $|F(x, u)| \leq \beta(|u|)+2 \tau C_{1}$. Thus, all the conditions of $g$ in Lemma 2.2 are satisfied. Therefore, there exists a solution $u \in W_{0}^{1, p}(\Omega)$ of (DS). We put $u_{1}:=u$ and consider the equation $-\tau\left(1+\tau k_{1}\right) \operatorname{div} a(u, \nabla u)+F(x, u)=\varphi_{1}=$ $\beta\left(u_{1}\right)+\tau C_{1} \operatorname{sign}(u)+\tau^{2} k_{i} \operatorname{div} a\left(u_{1}, \nabla u_{1}\right)$ where $F(x, u)=\beta(u)+\tau f(x, 2 \tau, u)+$ $\tau C_{1} \operatorname{sign}(u)$. Continuing this process, we have a solution $u_{i}$ of (6) for $i=$ $1,2, \ldots, N$ such that $u_{i} \in W_{0}^{1, p}(\Omega)(i=1,2, \ldots, N)$.

Next, we show the uniqueness of $u_{i}(i=1,2, \ldots, N)$. Let $u_{i}$ and $u_{i}^{*}$ be two solutions of (DS) for $i=1,2, \ldots, N$. Then we obtain that

$$
\begin{aligned}
& -\left(\tau+\tau^{2} k_{1}\right) \operatorname{div} a\left(u_{i}, \nabla u_{i}\right)+\left(\tau+\tau^{2} k_{1}\right) \operatorname{div} a\left(u_{i}^{*}, \nabla u_{i}^{*}\right)+\beta\left(u_{i}\right)-\beta\left(u_{i}^{*}\right) \\
& +\tau f\left(x, i \tau, u_{i}\right)-\tau f\left(x, i \tau, u_{i}^{*}\right)=0
\end{aligned}
$$


Multiplying (7) by $u_{i}-u_{i}^{*}$ and integrating over $\Omega$, gives

$$
\begin{aligned}
& \left\langle-\left(\tau+\tau^{2} k_{1}\right) \operatorname{div} a\left(u_{i}, \nabla u_{i}\right)+\left(\tau+\tau^{2} k_{1}\right) \operatorname{div} a\left(u_{i}^{*}, \nabla u_{i}^{*}\right), u_{i}-u_{i}^{*}\right\rangle \\
& +\int_{\Omega}\left\{\tau\left(f\left(x, i \tau, u_{i}\right)-f\left(x, i \tau, u_{i}^{*}\right)\right)+\left(\beta\left(u_{i}\right)-\beta\left(u_{i}^{*}\right)\right)\right\}\left(u_{i}-u_{i}^{*}\right) d x \\
& =0
\end{aligned}
$$

Then, from (H3), we have

$$
\int_{\Omega}\left(f\left(x, i \tau, u_{i}\right)-f\left(x, i \tau, u_{i}^{*}\right)\right)\left(u_{i}-u_{i}^{*}\right) d x \geq-C_{2} \int_{\Omega}\left(\beta\left(u_{i}\right)-\beta\left(u_{i}^{*}\right)\right)\left(u_{i}-u_{i}^{*}\right) d x .
$$

Applying the above inequality to $(8)$, by $(H 2)$,

$$
\left(1-\tau C_{2}\right) \int_{\Omega}\left(\beta\left(u_{i}\right)-\beta\left(u_{i}^{*}\right)\right)\left(u_{i}-u_{i}^{*}\right) d x \leq 0 .
$$

Then by (H1), if $\tau<1 / C_{2}$, we get $u_{i}=u_{i}^{*}$.

Now, we consider the bounds of $\left\{u_{i}\right\}(i=1,2, \ldots, N)$, which is constructed in Theorem 3.5 as solutions of (DS).

Theorem 3.6. Assume $(H 1)-(H 3)$ and $(H 6)$. Then there exist $C_{3}, C_{4}$, which are positive constants and independent of $\tau$, such that for all $i=1,2, \ldots, m$,

$$
\begin{aligned}
& \text { (a) } \tau \sum_{i=1}^{m}\left\|u_{i}\right\|_{1, p}^{p} \leq C_{3}, \\
& \text { (b) }\left\|\beta\left(u_{m}\right)\right\|_{2}^{2}+\sum_{i=1}^{m}\left\|\beta\left(u_{i}\right)-\beta\left(u_{i-1}\right)\right\|_{2}^{2} \leq C_{4},
\end{aligned}
$$

$m=1,2, \ldots, N$.

Proof. (a) Let $z \in W_{0}^{1, p}(\Omega)$ be fixed and multiplying the equation (DS) by $u_{i}-z$ and integrating over $\Omega$ and by (H2) and (H3), we obtain

$$
\begin{aligned}
& \left\langle\beta\left(u_{i}\right)-\beta\left(u_{i-1}\right), u_{i}\right\rangle-\left\langle\beta\left(u_{i}\right)-\beta\left(u_{i-1}\right), z\right\rangle+\alpha \tau\left\|u_{i}\right\|_{1, p}^{p} \\
& \leq \tau C_{1} \int_{\Omega}\left|u_{i}-z\right| d x+\tau \int_{\Omega} \gamma\left(\left|u_{i}\right|^{p-1}+\left|\nabla u_{i}\right|^{p-1}+l(x)\right)|\nabla z| d x \\
& \quad+\tau\left\|\left(k *_{\tau} \operatorname{div} a(u, \nabla u)\right)_{i}\right\|_{-1, p^{\prime}}|| u_{i}-z \|_{1, p} .
\end{aligned}
$$

Applying (9) to discrete Young's inequality,

$$
\begin{aligned}
& \left\langle\beta\left(u_{i}\right)-\beta\left(u_{i-1}\right), u_{i}\right\rangle-\left\langle\beta\left(u_{i}\right)-\beta\left(u_{i-1}\right), z\right\rangle+\alpha \tau\left\|u_{i}\right\|_{1, p}^{p} \\
& \leq \frac{\alpha \tau}{2}\left\|u_{i}\right\|_{1, p}^{p}+\tau C\left(\alpha, p,\|z\|_{1, p},\|l\|_{p^{\prime}}, \lambda_{1}, C_{1}, m(\Omega)\right) \\
& \quad+\tau\left(\frac{\alpha p}{2^{p+3}}\right)^{-\frac{p^{\prime}}{p}}\left(p^{\prime}\right)^{-1}\left\|\left(k *_{\tau} \operatorname{div} a(u, \nabla u)\right)_{i}\right\|_{-1, p^{\prime}}^{p^{\prime}}
\end{aligned}
$$




$$
\begin{aligned}
\leq & \frac{\alpha \tau}{2}\left\|u_{i}\right\|_{1, p}^{p}+\tau C\left(\alpha, p,\|z\|_{1, p},\|l\|_{p^{\prime}}, \lambda_{1}, C_{1}, m(\Omega)\right) \\
& +\tau C\left(p, \alpha,\|k\|_{L^{\infty}(0, T)}, \gamma, \lambda_{1}\right) \sum_{j=1}^{i} \tau\left\|u_{j}\right\|_{1, p}^{p}
\end{aligned}
$$

for $i=1,2, \ldots, m$ and for arbitrary $m=1,2, \ldots, N$. Since

$$
\int_{\Omega} \psi^{*}\left(\beta\left(u_{i}\right)\right)-\psi^{*}\left(\beta\left(u_{i-1}\right)\right) d x \leq \int_{\Omega}\left(\beta\left(u_{i}\right)-\beta\left(u_{i-1}\right)\right) u_{i} d x
$$

and by (10),

$$
\begin{aligned}
& \int_{\Omega} \psi^{*}\left(\beta\left(u_{i}\right)\right)-\psi^{*}\left(\beta\left(u_{i-1}\right)\right) d x-\left\langle\beta\left(u_{i}\right)-\beta\left(u_{i-1}\right), z\right\rangle+\frac{\alpha \tau}{2}\left\|u_{i}\right\|_{1, p}^{p} \\
& \leq \tau C_{5}+\tau C_{6} \sum_{j=1}^{i} \tau\left\|u_{j}\right\|_{1, p}^{p},
\end{aligned}
$$

for $i=1,2, \ldots, m$ and where $C_{5}=C\left(\alpha, p,\|z\|_{1, p},\|l\|_{p^{\prime}}, \lambda_{1}, C_{1}, m(\Omega)\right)$ and $C_{6}=$ $C\left(p, \alpha,\|k\|_{L^{\infty}(0, T)}, \gamma, \lambda_{1}\right)$. Then summing the above inequality with respect to $i=1,2, \ldots, m$,

$$
\begin{aligned}
& \int_{\Omega} \psi^{*}\left(\beta\left(u_{m}\right)\right) d x-\left\langle\beta\left(u_{m}\right), z\right\rangle+\frac{\alpha \tau}{2} \sum_{i=1}^{m}\left\|u_{i}\right\|_{1, p}^{p} \\
& \leq \int_{\Omega} \psi^{*}\left(\beta\left(u_{0}\right)\right) d x-\left\langle\beta\left(u_{0}\right), z\right\rangle+C_{5} T+\tau C_{6} \sum_{i=1}^{m} \sum_{j=1}^{i} \tau\left\|u_{j}\right\|_{1, p}^{p},
\end{aligned}
$$

for $m=1,2, \ldots, N$. By (11) and for arbitrary $\tau<\bar{\tau}=\alpha / 4 C_{6}$ and applying the discrete Gronwall lemma,

$$
\begin{aligned}
& \int_{\Omega} \psi^{*}\left(\beta\left(u_{m}\right)\right) d x-\left\langle\beta\left(u_{m}\right), z\right\rangle+\frac{\tau \alpha}{4} \sum_{i=1}^{m}\left\|u_{i}\right\|_{1, p}^{p} \\
& \leq C\left(\alpha, p,\|z\|_{1, p},\|l\|_{p^{\prime}}, \lambda_{1}, C_{1}, m(\Omega),\|k\|_{L^{\infty}(0, T)}, \gamma, T\right) .
\end{aligned}
$$

Hence by $\int_{\Omega} \psi^{*}\left(\beta\left(u_{i}\right)\right) d x-\left\langle\beta\left(u_{i}\right), z\right\rangle>-\infty, \tau \sum_{i=1}^{m}\left\|u_{i}\right\|_{1, p}^{p} \leq C_{3}$.

(b)From (5) and (DS), and since $\left(\rho *_{\tau} \delta v\right)_{i}=\left(\delta \rho *_{\tau} v\right)_{i}+\rho_{0} v_{i}-\rho_{i} v_{0}$,

$$
\begin{aligned}
& \beta\left(u_{i}\right)-\beta\left(u_{i-1}\right)-\tau \operatorname{div} a\left(u_{i}, \nabla u_{i}\right) \\
& =-\tau f_{i}+\tau\left(\rho *_{\tau} f\right)_{i}+\tau\left(\delta \rho *_{\tau} \beta(u)\right)_{i}+\tau \rho_{0} \beta\left(u_{i}\right)-\tau \rho_{i} \beta\left(u_{0}\right) .
\end{aligned}
$$

Multiplying the equation (12) by $\beta\left(u_{i}\right)$ and integrating over $\Omega$ and by (H2)and $\frac{1}{2}(a-b)^{2}+\frac{1}{2}(a)^{2}-\frac{1}{2}(b)^{2}=(a-b) a$,

$$
\begin{aligned}
& \left\|\beta\left(u_{i}\right)-\beta\left(u_{i-1}\right)\right\|_{2}^{2}+\left\|\beta\left(u_{i}\right)\right\|_{2}^{2}-\left\|\beta\left(u_{i-1}\right)\right\|_{2}^{2} \\
& \leq C_{1}^{2} \tau m(\Omega)+\tau\left(3+2\left|\rho_{0}\right|+\left|\bar{\rho}_{\tau}\right|_{L^{\infty}(0, T)}\right)\left\|\beta\left(u_{i}\right)\right\|_{2}^{2} \\
& \quad+\tau\left|\bar{\rho}_{\tau}\right|_{L^{\infty}(0, T)}\left\|\beta\left(u_{0}\right)\right\|_{2}^{2}+\tau\left\|\left(\rho *_{\tau} f\right)_{i}\right\|_{2}^{2}+\tau\left\|\left(\delta \rho *_{\tau} \beta(u)\right)_{i}\right\|_{2}^{2} .
\end{aligned}
$$


Then summing(13) with respect to $i=1,2, \ldots, m$,

$$
\begin{aligned}
& \sum_{i=1}^{m}\left\|\beta\left(u_{i}\right)-\beta\left(u_{i-1}\right)\right\|_{2}^{2}+\left\|\beta\left(u_{m}\right)\right\|_{2}^{2}-\left\|\beta\left(u_{0}\right)\right\|_{2}^{2} \\
& \leq C_{1}^{2} T m(\Omega)+\tau\left(3+2\left|\rho_{0}\right|+\left|\bar{\rho}_{\tau}\right|_{L^{\infty}(0, T)}\right) \sum_{i=1}^{m}\left\|\beta\left(u_{i}\right)\right\|_{2}^{2} \\
& \quad+\left|\bar{\rho}_{\tau}\right|_{L^{\infty}(0, T)}\left\|\beta\left(u_{0}\right)\right\|_{2}^{2} T+\tau \sum_{i=1}^{m}\left\|\left(\rho *_{\tau} f\right)_{i}\right\|_{2}^{2} \\
& \quad+\tau \sum_{i=1}^{m}\left\|\left(\delta \rho *_{\tau} \beta(u)\right)_{i}\right\|_{2}^{2},
\end{aligned}
$$

for arbitrary $m=1,2, \ldots, N$. Since $\tau \sum_{i=1}^{m}\left\|\left(\rho *_{\tau} f\right)_{i}\right\|_{2}^{2} \leq 3 T^{3}\left|\bar{\rho}_{\tau}\right|_{L^{\infty}(0, T)}^{2} C_{1}^{2} m(\Omega)$ and by Lemma 3.1, $\tau \sum_{i=1}^{m}\left\|\left(\delta \rho *_{\tau} \beta(u)\right)_{i}\right\|_{2}^{2} \leq m(\Omega)(\operatorname{Var}(\rho))^{2}\left(\sum_{i=1}^{m} \tau\left\|\beta\left(u_{i}\right)\right\|_{2}^{2}\right)$ and from (14),

$$
\sum_{i=1}^{m}\left\|\beta\left(u_{i}\right)-\beta\left(u_{i-1}\right)\right\|_{2}^{2}+\left\|\beta\left(u_{m}\right)\right\|_{2}^{2} \leq C_{7}+C_{8} \sum_{i=1}^{m} \tau\left\|\beta\left(u_{i}\right)\right\|_{2}^{2},
$$

for arbitrary $m=1,2, \ldots, N$, where $C_{7}=C\left(C_{1}, T, m(\Omega),\left|\bar{\rho}_{\tau}\right|_{L^{\infty}(0, T)}, \beta\left(u_{0}\right)\right)$ and $C_{8}=C\left(m(\Omega),\left|\bar{\rho}_{\tau}\right|_{L^{\infty}(0, T)},\left|\rho_{0}\right|, \operatorname{Var}(\rho)\right)$. By (15) and discrete Gronwall Lemma to the above inequality for arbitrary $\tau<\bar{\tau}=1 / 2 C_{8}$,

$$
\left\|\beta\left(u_{m}\right)\right\|_{2}^{2}+\sum_{i=1}^{m}\left\|\beta\left(u_{i}\right)-\beta\left(u_{i-1}\right)\right\|_{2}^{2} \leq C_{4} .
$$

Hence we are entitled to rewrite (DS) in a more compact form as

$$
\begin{aligned}
& v_{\tau}^{\prime}-\operatorname{div} a\left(\bar{u}_{\tau}, \nabla \bar{u}_{\tau}\right)-{\overline{\left(k *_{\tau} \operatorname{div} a(u, \nabla u)\right)_{\tau}}}+\bar{f}_{\tau}=0 \text { a.e. in }[0, T], \\
& \bar{v}_{\tau}=\beta\left(\bar{u}_{\tau}\right) \\
& \text { a.e. in }[0, T] \text {. }
\end{aligned}
$$

\section{Estimate and limits}

In this section, we assume the hypotheses (H1), (H2) and (H4)-(H6).

\subsection{Estimate}

First of all, by the consequence of Theorem 3.6, the followings are very easily proven.

$$
\begin{aligned}
& \bar{u}_{\tau} \text { is bounded in } L^{p}\left(0, T ; W_{0}^{1, p}(\Omega)\right), \\
& v_{\tau} \text { is bounded in } C\left(0, T ; L^{2}(\Omega)\right) .
\end{aligned}
$$

Moreover, by (17) and (H2),

$$
-\operatorname{div} a\left(\bar{u}_{\tau}, \nabla \bar{u}_{\tau}\right) \text { is bounded in } L^{p^{\prime}}\left(0, T ; W^{-1, p^{\prime}}(\Omega)\right) \text {. }
$$


We emphasis that all the above boundedness are independent of $\tau$. By (17), we have $u \in L^{p}\left(0, T ; W_{0}^{1, p}(\Omega)\right)$ which is a weak limit of $\bar{u}_{\tau}$ as $\tau \rightarrow 0$. i.e., $\bar{u}_{\tau}$ converges weakly to $u$ as $\tau \rightarrow 0$ in $L^{p}\left(0, T ; W_{0}^{1, p}(\Omega)\right)$. Moreover, by Theorem 3.6(b) we may use the term $w(x):=\sup _{t \in[0, T]} \frac{\partial \beta(u(x, t))}{\partial t}$ which is bounded in $L^{2}(\Omega)$ a.e.

Now, we consider the boundedness of $v_{\tau}^{\prime}$. By (H4) and Theorem 3.6(b),

$$
\begin{aligned}
& \sum_{i=1}^{N} \int_{(i-1) \tau}^{i \tau}\left\|f(x, t, u)-f\left(x, t, \bar{u}_{\tau}\right)\right\|_{-1, p^{\prime}}^{p^{\prime}} d t \\
& \left.\leq \sum_{i=1}^{N} \int_{(i-1) \tau\|v\|_{1, p \leq 1} \leq 1}^{i \tau}\left\{\int_{\Omega}|v(x)|^{p} d x\right)^{\frac{1}{p}}\left(\int_{\Omega}\left|f(x, t, u)-f\left(x, t, \bar{u}_{\tau}\right)\right|^{p^{\prime}} d x\right)^{\frac{1}{p^{\prime}}}\right\}^{p^{\prime}} d t \\
& \leq \lambda_{1}{ }^{p^{\prime}} C_{M} \tau \sum_{i=1}^{N} \int_{(i-1) \tau}^{i \tau}\left(\|u\|_{2}+\left\|\bar{u}_{\tau}\right\|_{2}\right)\|w\|_{2} d t \\
& \leq \lambda_{1}{ }^{p^{\prime}} C_{M} \tau\left(\|u\|_{L^{2}\left(0, T ; L^{2}(\Omega)\right)}+\left\|\bar{u}_{\tau}\right\|_{L^{2}\left(0, T ; L^{2}(\Omega)\right)}\right)\|w\|_{L^{2}\left(0, T ; L^{2}(\Omega)\right)} .
\end{aligned}
$$

By (H5),

$$
\begin{aligned}
& \sum_{i=1}^{N} \int_{(i-1) \tau}^{i \tau}\left\|f\left(x, t, \bar{u}_{\tau}(x, t)\right)-f\left(x, i \tau, \bar{u}_{\tau}(x, t)\right)\right\|_{-1, p^{\prime}}^{p^{\prime}} d t \\
& \leq \widetilde{C}_{M}^{p^{\prime}} \tau \sum_{i=1}^{N} \int_{(i-1) \tau}^{i \tau}\left[\sup _{\|v\|_{1, p} \leq 1}\left|\int_{\Omega}\right| v(x) \mid d x\right]^{1 / p^{\prime}} d t \\
& \leq \widetilde{C}_{M}^{p^{\prime}} \tau \sum_{i=1}^{N} \int_{(i-1) \tau}^{i \tau} \lambda_{4}^{p^{\prime}} d t=\widetilde{C}_{M}^{p^{\prime}} \tau \lambda_{4}^{p^{\prime}} T .
\end{aligned}
$$

Hence

$$
\begin{aligned}
& \left\|\bar{f}_{\tau}\left(x, t, \bar{u}_{\tau}\right)-f(x, t, u)\right\|_{L^{p^{\prime}}\left(0, T ; W^{-1, p^{\prime}}(\Omega)\right)}^{p^{\prime}} \\
& \leq 2^{p^{\prime}}\left(\lambda_{1}^{p^{\prime}} C_{M} \tau\left(\|u\|_{L^{2}\left(0, T ; L^{2}(\Omega)\right)}+\left\|\bar{u}_{\tau}\right\|_{L^{2}\left(0, T ; L^{2}(\Omega)\right)}\right)\|w\|_{L^{2}\left(0, T ; L^{2}(\Omega)\right)}\right. \\
& \left.+\widetilde{C}_{M}^{p^{\prime}} \tau \lambda_{4}^{p^{\prime}} T\right) .
\end{aligned}
$$

And by Corollary 3.3 and (19),

$$
-\overline{\left(k *_{\tau} \operatorname{div} a(u, \nabla u)\right)_{\tau}} \text { is bounded in } L^{p^{\prime}}\left(0, T ; W^{-1, p^{\prime}}(\Omega)\right) .
$$

Since $v_{\tau}^{\prime}=\operatorname{div} a\left(\bar{u}_{\tau}, \nabla \bar{u}_{\tau}\right)+{\overline{\left(k *_{\tau} \operatorname{div} a(u, \nabla u)\right)_{\tau}}}_{-\bar{f}_{\tau}}$ in (16), by (19)-(21), we conclude that

$$
v_{\tau}^{\prime} \text { is bounded in } L^{p^{\prime}}\left(0, T ; W^{-1, p^{\prime}}(\Omega)\right) .
$$




\subsection{Limits}

As we mentioned in $(17),(18),(20)$ and (22), and thanks to well-known compactness results (see [[16], Corollary 4]) we have $u, v$ and $f$ such that

$$
\begin{aligned}
& \bar{u}_{\tau} \rightarrow u \text { weakly in } L^{p}\left(0, T ; W_{0}^{1, p}(\Omega)\right), \\
& v_{\tau} \rightarrow v \text { weakly star in } W^{1, p^{\prime}}\left(0, T ; W^{-1, p^{\prime}}(\Omega)\right) \\
& v_{\tau} \rightarrow v \text { strongly in } C\left(0, T ; L^{2}(\Omega)\right) \\
& \bar{v}_{\tau} \rightarrow v \text { weakly star in } L^{p^{\prime}}\left(0, T ; W^{-1, p}(\Omega)\right) \\
& \bar{v}_{\tau} \rightarrow v \text { strongly in } L^{\infty}\left(0, T ; L^{2}(\Omega)\right) \\
& \bar{f}_{\tau} \rightarrow f \text { strongly in } L^{p^{\prime}}\left(0, T ; W^{-1, p^{\prime}}(\Omega)\right) .
\end{aligned}
$$

We note that the above sequences with $\tau$ are for some not relabeled subsequence. Also we note that

$$
\begin{aligned}
\lim _{\tau \rightarrow 0} \int_{0}^{T}\left\langle\phi, \bar{v}_{\tau}-v_{\tau}\right\rangle d t & \leq \lim _{\tau \rightarrow 0} \tau \sum_{i=1}^{N} \int_{(i-1) \tau}^{i \tau} \int_{\Omega} \phi(x, t)\left(\frac{v_{i}-v_{i-1}}{\tau}\right) d x d t \\
& =\lim _{\tau \rightarrow 0} \tau \sum_{i=1}^{N} \int_{(i-1) \tau}^{i \tau} \int_{\Omega} \phi(x, t) v_{\tau}^{\prime}(t) d x d t \\
& =\lim _{\tau \rightarrow 0} \tau \int_{0}^{T}\left\langle\phi, v_{\tau}^{\prime}\right\rangle d t=0
\end{aligned}
$$

for all $\phi \in L^{p}\left(0, T ; W_{0}^{1, p}(\Omega)\right)$ by $(22)$. Hence, $v_{\tau}$ and $\bar{v}_{\tau}$ have the same limit $v$ in (24) and (25). In addition, by (23), (25) and (H1), we have $v=\beta(u)$.

Proof of Theorem 2.3. It is enough to prove that Proof of Theorem 2.3. By (5) and $(16), \delta \bar{v}_{\tau}-\operatorname{div} a\left(\bar{u}_{\tau}, \nabla \bar{u}_{\tau}\right)=-\bar{f}_{\tau}+\left(\overline{\rho *_{\tau} f}\right)_{\tau}+\left(\overline{\rho *_{\tau} \delta v}\right)_{\tau}$ and then

$$
\begin{aligned}
& \lim \sup _{\tau \rightarrow 0} \int_{0}^{T}\left\langle-\operatorname{div} a\left(\bar{u}_{\tau}, \nabla \bar{u}_{\tau}\right), \bar{u}_{\tau}\right\rangle d t \\
& \leq \lim \sup _{\tau \rightarrow 0} \int_{0}^{T}\left\langle-\delta \bar{v}_{\tau}, \bar{u}_{\tau}\right\rangle d t+\lim \sup _{\tau \rightarrow 0} \int_{0}^{T}\left\langle-f_{\tau}+\left(\overline{\rho *_{\tau} f}\right)_{\tau}, \bar{u}_{\tau}\right\rangle d t \\
& \quad+\lim \sup _{\tau \rightarrow 0} \int_{0}^{T}\left\langle\left(\overline{\rho *_{\tau} \delta v}\right)_{\tau}, \bar{u}_{\tau}\right\rangle d t
\end{aligned}
$$

In the equation (27),

$$
\begin{aligned}
& \lim \sup _{\tau \rightarrow 0} \int_{0}^{T}\left\langle-\delta \bar{v}_{\tau}, \bar{u}_{\tau}\right\rangle d t \\
& =\lim \sup _{\tau \rightarrow 0} \sum_{i=1}^{N} \int_{(i-1) \tau}^{i \tau} \int_{\Omega}-\frac{\beta\left(u_{i}\right)-\beta\left(u_{i-1}\right)}{\tau} u_{i} d x d t .
\end{aligned}
$$


Since $\int_{\Omega} \psi^{*}\left(\beta\left(u_{i}\right)\right)-\psi^{*}\left(\beta\left(u_{i-1}\right)\right) d x \leq \int_{\Omega}\left(\beta\left(u_{i}\right)-\beta\left(u_{i-1}\right)\right) u_{i} d x$,

$$
\begin{aligned}
& \lim \sup _{\tau \rightarrow 0} \int_{0}^{T}\left\langle-\delta \bar{v}_{\tau}, \bar{u}_{\tau}\right\rangle d t \\
& \leq \lim \sup _{\tau \rightarrow 0} \sum_{i=1}^{N} \int_{(i-1) \tau}^{i \tau} \int_{\Omega} \frac{-\psi^{*}\left(\beta\left(u_{i}\right)\right)+\psi^{*}\left(\beta\left(u_{i-1}\right)\right)}{\tau} d x d t .
\end{aligned}
$$

Since $\psi^{*}(\beta(u(i \tau)))-\psi^{*}(\beta(u((i-1) \tau)))=\frac{\partial \psi^{*}}{\partial s}(\beta(u(\sigma)))(i \tau-(i-1) \tau), \sigma \in$ $((i-1) \tau, i \tau]$,

$$
\begin{aligned}
& \lim \sup _{\tau \rightarrow 0} \int_{0}^{T}\left\langle-\delta \bar{v}_{\tau}, \bar{u}_{\tau}\right\rangle d t \\
& \leq \lim \sup _{\tau \rightarrow 0} \sum_{i=1}^{N} \int_{(i-1) \tau}^{i \tau} \frac{1}{\tau} \int_{\Omega}-\tau \frac{\partial \psi^{*}(\beta(u(s)))}{\partial s} d x d t \\
& =\int_{0}^{T} \int_{\Omega}-\frac{\partial \psi^{*}(\beta(u(s)))}{\partial s} d x d t .
\end{aligned}
$$

Since $\psi(t)=\int_{0}^{t} \beta(s) d s, \psi^{\prime}(t)=\beta(t)$ and $\left(\psi^{*}\right)^{\prime}=\left(\psi^{\prime}\right)^{-1}$,

$$
\begin{aligned}
\lim \sup _{\tau \rightarrow 0} \int_{0}^{T}\left\langle-\delta \bar{v}_{\tau}, \bar{u}_{\tau}\right\rangle d t & \leq \int_{0}^{T} \int_{\Omega}-\frac{\partial \beta(u(s))}{\partial s} u(s) d x d t \\
& =\int_{0}^{T}\left\langle-\frac{\partial \beta(u)}{\partial t}, u\right\rangle d t .
\end{aligned}
$$

As for the terms containing ${\overline{\rho *_{\tau} \delta v_{\tau}}}_{\text {in }}(27)$, we exploit Proposition 3.2 and obtain

$$
{\overline{\rho *_{\tau} \delta v_{\tau}}}^{\longrightarrow} \bar{\rho}_{\tau} * v_{\tau}^{\prime} \text { strongly in } L^{p^{\prime}}\left(0, T ; W^{-1, p^{\prime}}(\Omega)\right) .
$$

On the other hand, by recalling that $\rho_{\tau}(0)=k(0)$ one readily computes that

$$
\begin{aligned}
\bar{\rho}_{\tau} * v_{\tau}^{\prime} & =\rho_{\tau} * v_{\tau}^{\prime}+\left(\bar{\rho}_{\tau}-\rho_{\tau}\right) * v_{\tau}^{\prime} \\
& =\rho_{\tau}^{\prime} * v_{\tau}+k(0) v_{\tau}-\rho_{\tau} v_{0}+\left(\bar{\rho}_{\tau}-\rho_{\tau}\right) * v_{\tau}^{\prime} \\
& =\rho_{\tau} * v^{\prime}+k(0)\left(v_{\tau}-v\right)+\rho_{\tau}^{\prime} *\left(v_{\tau}-v\right)+\left(\bar{\rho}_{\tau}-\rho_{\tau}\right) * v_{\tau}^{\prime} .
\end{aligned}
$$

By recalling Proposition 3.4 and (24), it is standard matter to check that the above right-hand side converges strongly to $\rho * v^{\prime}$ in $L^{p^{\prime}}\left(0, T ; W^{-1, p^{\prime}}(\Omega)\right)$. Therefore, we readily conclude that

$$
{\overline{\rho *_{\tau} \delta v_{\tau}}} \longrightarrow \rho * v^{\prime} \text { strongly in } L^{p^{\prime}}\left(0, T ; W^{-1, p^{\prime}}(\Omega)\right)
$$

By Corollary 3.3 and Proposition 3.4, we check that

$$
-\bar{f}_{\tau}+{\overline{\left(\rho *_{\tau} f\right)_{\tau}}}^{\longrightarrow}-f+\rho * f \text { strongly in } L^{p^{\prime}}\left(0, T ; W^{-1, p^{\prime}}(\Omega)\right) .
$$

Applying (28),(29),(30) to (27) and by Lemma 2.1 (Minty's Theorem), there exist a solution $u \in L^{p}\left(0, T ; W^{1, p}(\Omega)\right)$ of (1) and initial condition is satisfied. 


\section{References}

[1] R. Adams, Sovolev Spaces, Academic Press, New York, 1975.

[2] Alt, W. Hans and S. Luckhaus, Qusilinear elliptic-parabolic differential equations, Math. Z. 83 (1983), 311-341.

[3] V. Barbu, Nonlinear semigroups and differential equations in Banach spaces, Noordhoff Internat. Publ. Leyden, 1976.

[4] A. Bensoussan, A. L. Boccardo and F. Murat, On a nonlinear P.D.E. having natural growth terms and unbounded solutions, Ann. Inst. H. Poincare 5 (1988), no. 4, 347-364.

[5] J. I. Diaz, and J. F. Padial, Uniqueness and existence of solutions in the $B V_{t}(Q)$ space to a doubly nonlinear parabolic problem, Differential and Integral Eqns. 40 (1966), 527560 .

[6] A. Eden, B. Michaux and J. M. Rakotoson, Semidiscretized nonlinear evolution equations as discrete dynamical systems and error analysis, Indiana Univ. Math. J. 39 (1990), no. 3, 737-783.

[7] _ Doubly nonlinear parabolic type equations as dynamical systems, J. Dynam. Diff. Equ. 3 (1991), no. 1, 87-131.

[8] A. El Hachimi and H. El Ouardi, Existence and regularity of a global attractor for doubly nonlinear parabolic equations, Electro. J. Diff. Equ. 2002 (2002), no. 45, 1-15.

[9] G. Gilardi and U. Stefanelli, Time-discretization and global solution for a doubly nonlinear Volterra equation, J. Diff. Equ. 228 (2006), 707-736.

[10] A. V. Ivanov and J. F. Rodrigues, Existence and uniqueness of a weak solution to the initial mixed boundary-value problem for Quasilinear elliptic-parabolic equations, J. Math. Sci. 109 (2002), no. 5, 1851-1866.

[11] F. Otto, $L^{1}$-contraction and uniqueness for Quasilinear Elliptic-Parabolic equations, J. Diff. Equ. 131 (1996), 20-38.

[12] A. Rougirel, Convergence to steady state and attractors for doubly nonlinear equations, J. Math. Anal. Appl. 339 (2008), 281-294.

[13] M. Schatzman, Stationary solutions and asymptotic behavior of a Quasilinear degenerate parabolic equation, Indiana Univ. Math. J. 33 (1984), no. 1, 1-29.

[14] K. Shin and S. Kang, Doubly nonlinear parabolic equations related to the leray-lions operators:time-discretization, East Asian Math. J. 26 (2010), no. 3, 403-413.

[15] R. E. Showalter, Monotone operators in Banach space and nonlinear partial differential equations, Math. Surveys and Monographs 49, Amer. Math. Soc.

[16] J. Simon, Compact sets in the space $L^{p}(0, T: B)$, Ann. Math. Pure and Appl. 146 (1987), no. 4, 65-96.

[17] U. Stefanelli, Well-posedness and time discretization of a nonlinear Volterra integrodiffrential equation, J. Integral Eqns. Appl. 13 (2001), no. 3, 273-304.

[18] $\ldots$ On some nonlocal evolution equation in Banach space, J. Evol. Eqns. 4 (2004), $1-26$.

KIYEON SHIN

Department of Mathematics, Pusan National University Pusan 609-735, Korea

E-mail address: kyshin@pusan.ac.kr

SUJIN KANG

Department of Nanomaterials Engineering, Pusan National University Pusan, 609-735, KOREA

E-mail address: sjnisj@pusan.ac.kr 